\title{
Spatial Analysis of Arabidopsis thaliana Gene Expression in Response to Turnip mosaic virus Infection
}

\author{
Chunling Yang, ${ }^{1}$ Rong Guo, ${ }^{2}$ Fei Jie, ${ }^{2}$ Dan Nettleton, ${ }^{2}$ Jiqing Peng, ${ }^{3}$ Tyrell Carr, ${ }^{1}$ Joanne M. Yeakley, ${ }^{4}$ \\ Jian-Bing Fan, ${ }^{4}$ and Steven A. Whitham ${ }^{1}$ \\ ${ }^{1}$ Department of Plant Pathology, ${ }^{2}$ Department of Statistics, and ${ }^{3}$ GeneChip Facility, lowa State University, Ames 50011 \\ U.S.A.; ${ }^{4}$ Illumina, Incorporated, 9885 Towne Centre Drive, San Diego, CA 92121, U.S.A.
}

Submitted 2 October 2006. Accepted 27 October 2006.

\begin{abstract}
Virus-infected leaf tissues comprise a heterogeneous mixture of cells at different stages of infection. The spatial and temporal relationships between sites of virus accumulation and the accompanying host responses, such as altered host gene expression, are not well defined. To address this issue, we utilized Turnip mosaic virus (TuMV) tagged with the green fluorescent protein to guide the dissection of infection foci into four distinct zones. The abundance of Arabidopsis thaliana mRNA transcripts in each of the four zones then was assayed using the Arabidopsis ATH1 GeneChip oligonucleotide microarray (Affymetrix). mRNA transcripts with significantly altered expression profiles were determined across gradients of virus accumulation spanning groups of cells in and around foci at different stages of infection. The extent to which TuMV-responsive genes were up- or downregulated primarily correlated with the amount of virus accumulation regardless of gene function. The spatial analysis also allowed new suites of coordinately regulated genes to be identified that are associated with chloroplast functions (decreased), sulfate assimilation (decreased), cell wall extensibility (decreased), and protein synthesis and turnover (induced). The functions of these downregulated genes are consistent with viral symptoms, such as chlorosis and stunted growth, providing new insight into mechanisms of pathogenesis.
\end{abstract}

Additional keywords: Potyvirus, ribosomal protein.

Viral pathogenesis is accompanied by a variety of changes in plant gene expression that likely reflect the ways that viruses generically and specifically interact with host cells (Golem and Culver 2003; Huang et al. 2005; Lecellier and Voinnet 2004; Maule et al. 2002; Whitham and Wang 2004; Whitham et al. 2003, 2006). Altered expression of some host genes may be required for basic compatibility, such as the induction of proliferating cell nuclear antigen by Geminivirus replication proteins (Hanley-Bowdoin et al. 2000; Nagar et al. 1995). Other gene expression changes, such as those caused by interference with miRNA functions, may be coin-

Corresponding author: S. A. Whitham; Telephone: +1.515.294.4952; Fax: +1.515.294.9420; E-mail: swhitham@iastate.edu

Arabidopsis ATH1 microarray data is available in the ArrayExpress database under accession number e-mexp-509.

* The $e$-Xtra logo stands for "electronic extra" and indicates the HTML abstract available online contains supplemental material not included in the print edition. Three supplemental tables appear online. cidental because viruses invade rapidly growing tissues and cause characteristic changes in plant phenotype (i.e., disease symptoms) (Chapman et al. 2004; Chen et al. 2004; Dunoyer et al. 2004). Recently, it has become clear that viral proteins also contribute to altered host gene expression and symptoms in ways that are independent of miRNA function. For example, certain plant viruses can interfere specifically with auxin signaling or giberellin accumulation (Padmanabhan et al. 2005, Zhu et al. 2005). At the present time, it is unclear whether interference with the function or activity of these hormones is coincidental or whether the hormones are important to specific viral infection processes.

In addition to specific responses to viruses, some studies have shown that there are general responses to different viruses that occur in susceptible hosts (Whitham et al. 2006). Generic gene expression changes include induction of defense-related genes, such as pathogenesis-related (PR) genes and heat shock (HS) genes (Whitham et al. 2003). Increased expression of defense-related genes, such as $P R-1$ and BGL2 ( $\beta$-1,3-glucanase $2)$, requires salicylic acid (SA), demonstrating that compatible host-virus interactions elicit a key defense signaling pathway (Huang et al. 2005). Viral infection also induces the expression of a discrete set of HS genes, which are a subset of those that are induced during heat stress. The induction of HS genes, including $H S P 70$, may be a general response to unfolded or misfolded viral proteins as they accumulate and aggregate in the cytoplasm (Aparicio et al. 2005). The nature of genes that are repressed and the extent to which they become downregulated by plant virus infection is relatively unexplored, although it is known to occur (Wang and Maule 1995).

Analyses of host-pathogen interactions are complicated by the fact that infected leaves comprise a heterogeneous mixture of host responses as pathogens spread from the primary inoculated cells. In the case of viruses, this heterogeneity occurs because infection is a continuous process beginning with virions entering a plant cell, followed by propagation of viral progeny and local movement to adjacent cells. The radial pattern of movement typical of many viruses results in foci containing a continuum of cells at different stages of infection. Cells at the center of foci have been colonized the longest time, the most recently infected cells are at the periphery, and the surrounding cells are not yet infected but already may be under the influence of a rapidly spreading virus (Maule et al. 2002). Within these foci, viral RNA and protein products accumulate, often to extreme levels relative to most cellular RNAs and proteins, and influence the host as they perform the variety of functions needed for successful infection (Carrington and Whitham 1998; Lecellier and Voinnet 2004; Maule et al. 2002; Voinnet 2001; Whitham and Wang 2004). 
The progressive and asynchronous effects of viral infection on host gene expression have been investigated previously by in situ hybridization. These studies correlated sites of accumulation of both (+) and (-) strands of viruses with host gene expression changes occurring in pea and squash cotyledons (Aranda et al. 1996; Escaler et al. 2000; Maule et al. 2002; Wang and Maule 1995). Predictable responses were associated with viral infection, such as the induction of HSP70 as well as the suppression of host genes. In pea and squash cotyledon systems, most of these changes occurred at sites containing the most active viral replication and moved as a wave across the cotyledon along with the virus replication front. Though these studies have provided exquisite resolution at the cellular level, they could not provide a global picture of host gene expression occurring in true leaves. Furthermore, the complexity of host responses is compounded by systemic movement, as the virus life cycle repeats itself in various plant tissues spanning a developmental gradient, which cannot be tested in the cotyledon system.

Here, we explored the altered expression profiles of host genes in relation to the spatial and temporal distribution of Turnip mosaic virus (TuMV; genus Potyvirus) infection sites in leaves of Arabidopsis thaliana plants and identified new suites of host genes with altered expression profiles in response to infection. TuMV was selected for these studies because of its pathogenicity in $A$. thaliana, the availability of an infectious clone expressing green fluorescent protein (GFP), and our previous data on its effects on host gene expression (Whitham et al. 2003). In order to obtain spatial and temporal information, TuMV tagged with GFP (TuMV-GFP) was used to provide a nondestructive guide to dissect infected leaf tissue away from noninfected tissue. TuMV-GFP is a well-characterized recombinant virus that causes symptoms in susceptible A. thaliana ecotypes and is readily traceable in the presence of ultraviolet (UV) illumination (Lellis et al. 2002; Sanchez et al. 1998). This strategy provided a higher-resolution picture of how host leaf cells respond to local infection across a spatial gradient. Using this approach, new groups of co-regulated genes were identified that are expected to have roles in viral pathogenesis, and new insight into the spatial and temporal relationships of viral infection and host gene expression was obtained.

\section{RESULTS}

\section{Dissection of TuMV-GFP infection foci.}

Considering the natural processes of viral replication and spread away from the initial inoculation sites, we hypothesized that host gene expression was not modified uniformly in infected leaves, but that there were spatial relationships between sites of virus accumulation and altered accumulation of host mRNA transcripts. To test this hypothesis, TuMV-GFP infection foci were dissected into four zones that separated virusinfected tissue from noninfected tissue in inoculated leaves. In order to optimize the dissection strategy, a time course was established for expansion of GFP-fluorescent foci. Infection foci were well defined and expanded in a predictable manner in inoculated rosette leaves of Col-0 plants (Fig. 1A and B). The growth in area of the foci was approximately linear with respect to time from 72 to $120 \mathrm{~h}$ after infection (hai) (Fig. 1B), and they continued to expand until becoming confluent due to extensive local and systemic movement by 168 hai. The 120 hai timepoint was chosen because the foci were expanding rapidly and their diameters were most ideal for the dissection strategy represented schematically in Figure 1C. Four zones were defined based on the size of typical foci (approximately 4 $\mathrm{mm}$ in diameter) and the $1.2-\mathrm{mm}$ diameter Harris micropunch that was used for dissection. The cross section of the micro- punch was estimated to be 26 to 30 epidermal cells in diameter, which is consistent with previous reports (Melaragno et al. 1993). Zone 0 was at the epicenter and the adjacent zone 1 included the remaining area of most intense GFP fluorescence. Zone 2 was at the periphery of the infection foci and possessed relatively weak GFP fluorescence, whereas zone 3 was distal and has no direct contact to tissue displaying GFP fluorescence.

According to the experimental procedure outlined in Figure $1 \mathrm{D}$, seven to eight fully expanded leaves on each of five Col-0
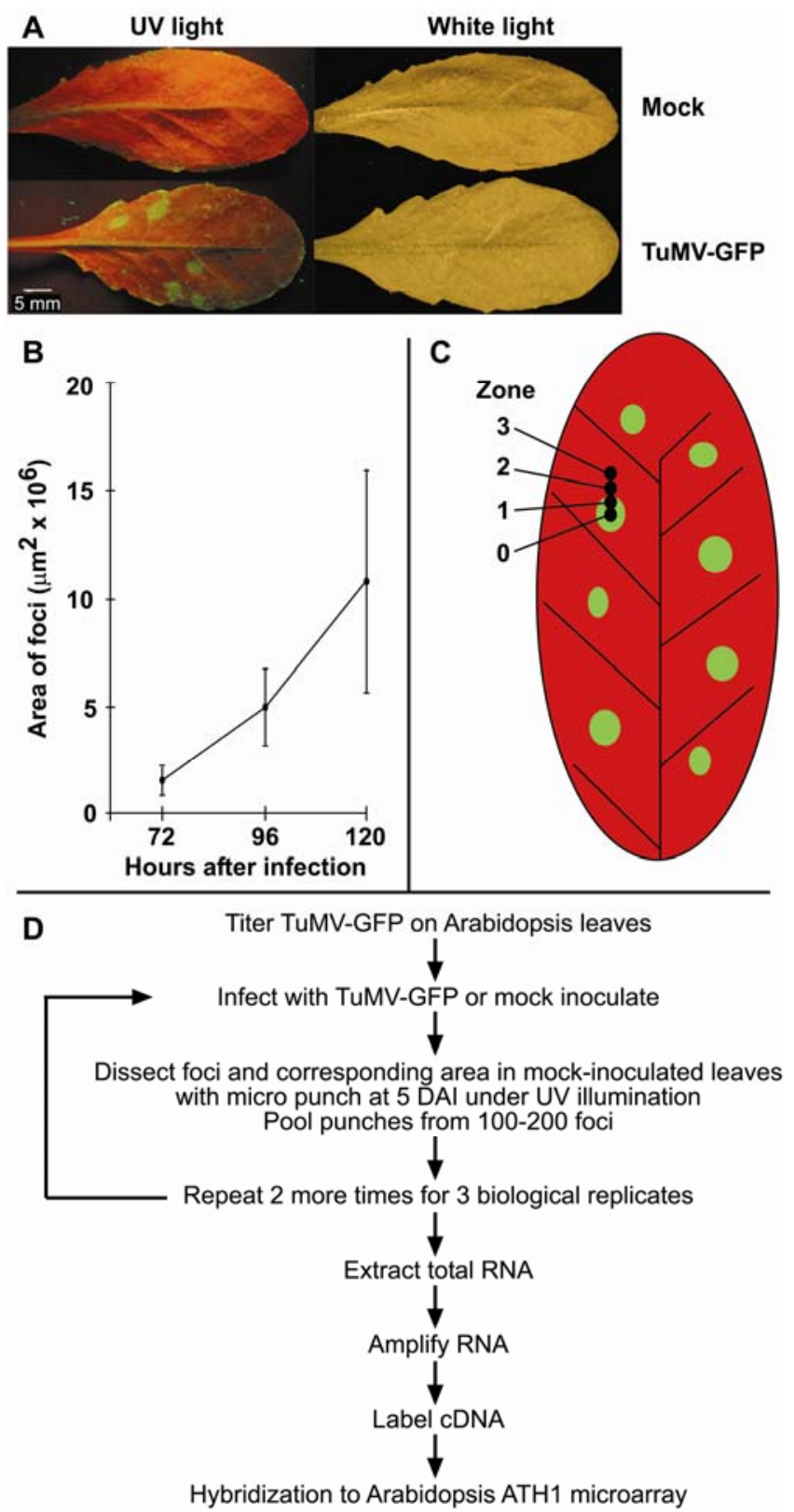

Fig. 1. Strategy for dissecting Turnip mosaic virus-green fluorescent protein (TuMV-GFP) infection foci for spatial analysis of host gene expression. A, Example of GFP-fluorescent infection foci in inoculated leaves at $120 \mathrm{~h}$ after infection (hai). Mock- and TuMV-GFP-inoculated leaves were photographed under illumination by ultraviolet (UV) (left panels) and white (right panels) light. B, The average area of TuMV-GFP infection foci \pm standard deviation at $72(n=16), 96(n=16)$, and $120(n=13)$ hai increased linearly with respect to time. Randomly selected TuMV-GFP foci were visualized by fluorescence microscopy and the area of each was determined by AxioVision software (Carl Zeiss MicroImaging, Inc, Thornwood NY, U.S.A.). C, Schematic representation for dissecting infection foci into four zones at 120 hai using a 1.2-mm Harris punch under illumination by UV light. D, Experimental design and work flow. 
plants were inoculated with TuMV-GFP and the corresponding leaves on another five plants were mock inoculated. At 120 hai, a pair of TuMV-GFP-infected and mock-inoculated plants was chosen randomly, and individual leaves were removed and exposed to UV light for dissection of foci. After sampling a leaf from a virus-infected plant, the corresponding areas of a matching mock-inoculated leaf were dissected under UV illumination. This procedure was reiterated until all inoculated leaves were sampled from each pair of plants. Approximately, 100 to 200 punches were pooled for each of the eight combinations of zone and treatment within each of three biological replicates that were inoculated at 1-day intervals. RNA was extracted from the 24 pools of leaf punches for amplification, labeling, and hybridization to Arabidopsis ATH1 genome arrays (Redman et al. 2004).

\section{Gene expression changes}

in relation to the accumulation of TuMV.

Strand-specific reverse-transcriptase polymerase chain reaction (RT-PCR) was used to determine the distribution of $(+)$

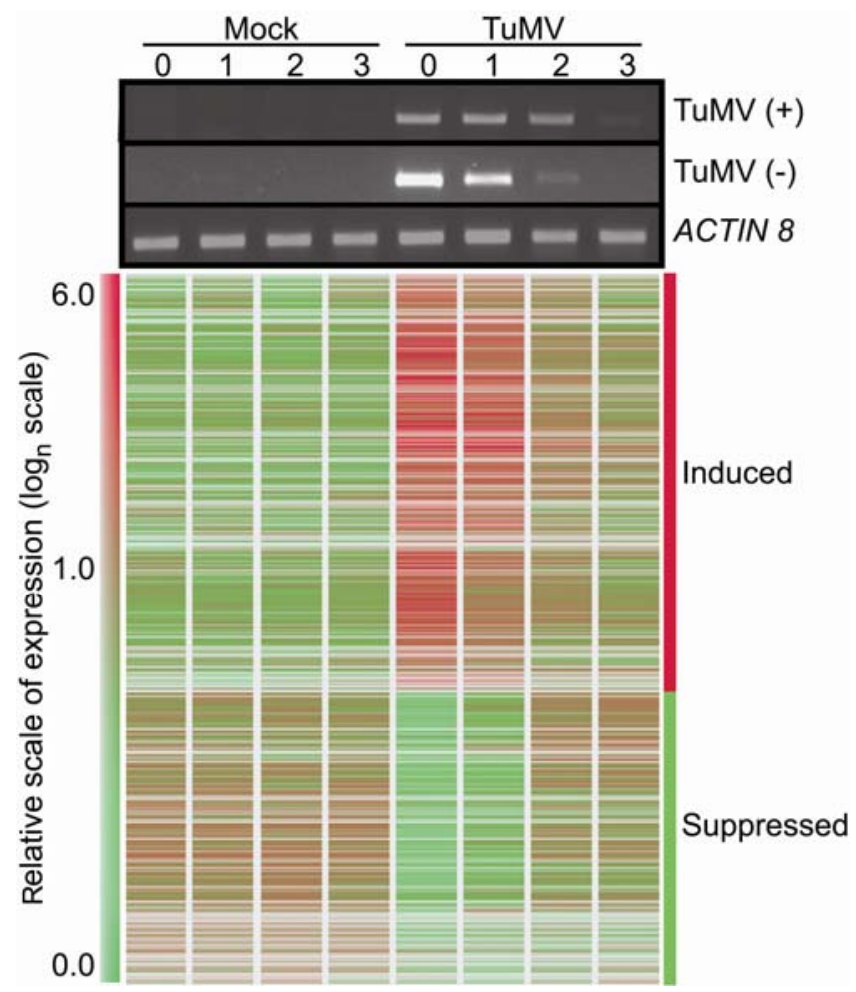

Fig. 2. Spatial relationships between accumulation of viral RNAs and altered expression of host genes. Reverse-transcriptase polymerase chain reaction was used to determine the distribution of (+)- and (-)-sense Turnip mosaic virus (TuMV) RNAs across zones 0, 1, 2, and 3 (upper panel). Primers were used to amplify the TuMV coat protein region, and Arabidopsis thaliana ACTIN 8 was used as a reference, because its expression was not significantly affected by TuMV infection. The lower panel shows the expression profiles of 556 genes that had significantly altered expression profiles $(q<0.05)$ across the four zones. The hierarchical clustering analysis delineates the 330 induced genes from the 226 downregulated genes. The average signal intensities for each of the 556 genes were loaded in GeneSpring (version 7.2; Agilent Technologies, Palo Alto, CA, U.S.A.) and standardized by median centering, and the similarities of expression profiles were calculated using a Standard correlation and the hierarchal clustering algorithm. The scale bar at the left side shows the natural log of the median-centered, relative expression level from low (green) to high (magenta). Green represents expression values less than the median $(<1.0)$, gray represents the median expression level $(\approx 1.0)$, and magenta represents expression values greater than the median $(>1.0)$. The intensity of the green or magenta represents the magnitude that the expression levels were below or above the median, respectively. and (-) sense TuMV RNA species across the four zones (Fig. 2, upper panel). TuMV (+)-strand RNA was most abundant in the center of the foci (zone 0 ) and its levels decreased in each of the successive zones, with a dramatic drop off from zone 2 to 3. The accumulation of (-)-strand RNA indicates sites where active replication is occurring (Wang and Maule 1995), and it was most abundant in zone 0 and became undetectable in zone 3 . These results demonstrate that TuMV replication was active in a decreasing gradient across zones 0,1 , and 2 , but not yet occurring in zone 3. The amplification of the TuMV strands is shown relative to ACTIN8, which is not altered in its expression profile during TuMV infection (data not shown).

We were most interested in gene expression changes caused by virus treatment or the interaction of virus treatment and zone. The effects due to zone alone were not considered here, because of possible complications resulting from the sampling method, such as rapid wound responses. The signal intensities for each gene were obtained from Microarray Analysis Suite 5.0 (Affymetrix, Santa Clara, CA, U.S.A.) and transformed by the natural logarithm. The data then were analyzed by a mixed model (Chu et al. 2002; Wolfinger et al. 2001) that used the Kenward-Roger method to determine denominator degrees of freedom (Kenward and Roger 1997) (discussed below). At the $5 \%$ false discovery rate (FDR) threshold $(q<0.05), 453$ genes were identified as differentially expressed between TuMV and the mock treatment when averaging over the zones, and 188 genes were identified as exhibiting patterns of expression across zones that differed significantly between treatments. In total, 556 unique genes with significantly altered expression profiles were identified.

Results of hierarchical cluster analysis of these 556 genes in relation to accumulation of (+)- and (-)-strand TuMV RNA in each zone are shown in the lower panel of Figure 2. The genes fall into two distinct categories, induced or suppressed, containing 330 and 226 members, respectively. For both categories, the altered expression levels are most extensive in zones 0 and 1 , with very few of them differing significantly from the mock-inoculated control in zone 3 , in which the TuMV (-)strand was not detectable. This observation indicates that most gene expression changes are localized and directly dependent upon the accumulation of TuMV. The expression profiles of the significant genes were relatively constant across the four zones in the mock-inoculated leaves, demonstrating that the genes selected by this analysis were altered due to the presence of viral infection and not because of the experimental procedures. A few genes appeared to have relatively constant expression across both mock-inoculated and TuMV-infected zones, indicating that their expression did not change dramatically; nevertheless, they met our statistical criteria.

\section{Functional classification of genes} with altered expression in response to TuMV-GFP infection.

To gain insight into the functions of genes with altered expression profiles in response to TuMV-GFP infection, the induced and suppressed gene sets were classified according to the Gene Ontology (GO) cellular component (Berardini et al. 2004) and the Munich Information Center for Protein Sequences (MIPS) functional classification (Schoof et al. 2004) (Fig. 3A and $\mathrm{B}$, respectively). The $\mathrm{GO}$ cellular component indicates where in a cell a gene product is known or suspected to localize and the MIPS functional class indicates its known or suspected function. We used the MIPS functional class instead of the GO functional class to be consistent with our previous work (Huang et al. 2005; Whitham et al. 2003). As expected, genes representing a spectrum of predicted functions and cellular locations were identified for both the induced and down- 
regulated gene sets. The proportions of induced or downregulated genes were decidedly skewed in some functional classes or cellular locations. For example, downregulated genes known or predicted to have cell wall and extracellular localization were significantly overrepresented when compared with the induced gene set (Fig. 3A). Genes encoding proteins predicted to reside in these locations include such known or suspected cell wall modification enzymes as xyloglucan endotransglycosylase/hydrolases (XTH6; At5g65730), pectinesterase (PME3, At3g14310), and expansin (EXP10, At1g26770). These genes, constitute a suite of cell-wall-modification genes whose expression levels dramatically decreased in response to TuMV infection. The chloroplast is another cellular compartment in which the downregulated genes are overrepresented. These genes include proteins with functions in light harvesting, photosynthesis, sulfur assimilation, and starch metabolism. In concordance with this observation is the fact that the MIPS function metabolism is composed largely of downregulated genes (30 genes) (Fig. 3B), of which 11 are predicted to encode proteins localized to the chloroplast.

The identification of downregulated genes involved in sulfur assimilation in the chloroplast led us to further investigate the effects of TuMV on the expression of genes involved in both sulfur assimilation and utilization. Interestingly, genes along the entire pathway from sulfur uptake to incorporation into defensive compounds, such as methionine-derived glucosinolates, are affected by TuMV infection. Beginning with sulfate uptake, both low-affinity (SULTR2;1, At5g10180) and high-affinity (SULTR4;2, At3g12520) transporters are downregulated. Genes that are predicted to function downstream of the transporters that assimilate inorganic sulfate into the amino acids cysteine and methionine are downregulated, including APS1 (At3g22890; ATP sulfurylase), APRI (At4g04610; APS reductase), a cysteine synthase homolog (At5g28020), and a gene encoding a key enzyme in the biosynthesis of methionine, cystathionine $\beta$-lyase. Continuing along the pathway to methioninederived glucosinolates, decreased expression also is observed for MAM1 (At5g23010), which catalyzes the first committed step in the synthesis of homomethoinine, and at least two genes (At4g13770, CYP83A1 monooxygenase and At4g03060, 2-oxoglutarate-dependent dioxygenase) involved in the synthesis of 2-propenyl-glucosinolate and 3-benzoyloxypropyl-glucosinolate from homomethionine. These results indicate that TuMV interferes with the ability of A. thaliana plants to acquire and utilize sulfur, which would have deleterious effects on plant growth, development, and defense.

Consistent with previous data (Whitham et al. 2003), the induced genes are overrepresented in the category of cell rescue, defense, and virulence, including HS genes and defenserelated genes (Fig. 3B). Strikingly, two new functional classes of induced genes were identified that are involved in protein synthesis and protein fate (Fig. 3B). With regard to protein synthesis, nearly one-quarter ( 80 of the 330 ) of the induced genes encode proteins assigned to the ribosome (Fig. 3A). The ribosome-associated proteins correspond to all the genes in the protein synthesis MIPS functional class (Fig. 3B). The overrepresentation of protein synthesis genes in the induced gene set was highly significant $(P<0.0001$ using Fisher's exact test (Fisher 1970). In all, 69 of the protein synthesis genes are associated with ribosome biogenesis, with most being constituents of either the $60 \mathrm{~S}$ or $40 \mathrm{~S}$ ribosomal subunits. The increased expression of these ribosomal proteins (RPs) demonstrates that their induction is coordinated in response to TuMV infection. The overrepresentation of protein fate genes, which comprise $10 \%$ of the induced gene set, also was determined to be highly significant $(P<0.0001$ using Fisher's exact test (Fisher 1970). These 32 genes encode proteins assigned to functions including protein targeting, sorting, and translocation (6 genes), assembly of protein complexes (11 genes), and protein degradation (14 genes). The genes associated with protein degradation encode seven subunits of the $20 \mathrm{~S}$ core proteasome, UMP1 (a chaperone required for $20 \mathrm{~S}$ proteasome assembly), two AAA-ATPases that reside in the mature $26 \mathrm{~S}$ proteasome, and four genes involved in ubiquitination. The ubiquitination genes encode polyubiquitin, ubiquitin extension protein, ubiquitin conjugating-like protein, and an E1 ubiquitin-like activating protein. These data suggest that a suite of genes that control the balance of cellular protein synthesis and fate are upregulated in response to TuMV.

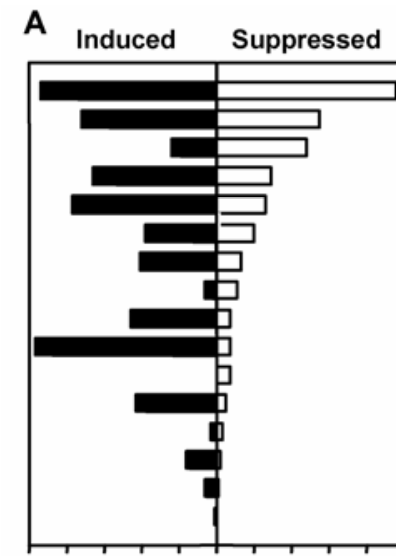

GO cellular component
Other membrane
Cellular component unknown
Chloroplast
Other cellular components
Other intracellular components
Mitochondria
Other cytoplasmic components
Extracellular
Nucleus
Ribosome
Cell wall
Cytosol
Plastid
Endoplasmic reticulum
Plasma membrane
Golgi apparatus

10080604020020406080100 Number of genes B Induced Suppressed

MIPS functional classification
Metabolism
Energy
Storage protein
Cell cycle and DNA processing
Transcription
Protein synthesis
Protein fate
Protein with binding function
Protein activity regulation
Cellular transport
Cellular communication
Cell rescue, defense and virulence
Interaction with cellular environment
Interaction with environment
Biogenesis of cellular components
Subcellular localization
Cell fate
Development
Cell type differentiation
Organ differentiation
Cell type localization
Tissue localization
Organ localization
Ubiquitous expression
Classification not yet clear-cut
Unclassified

Fig. 3. Classification of 556 differentially expressed genes according to cellular localization or function. The induced and repressed gene sets were classified according to $\mathbf{A}$, the known or predicted locations of their gene products within the cell (cellular component) using the TAIR Gene Ontology (GO) terms or B, the known or predicted functions of their encoded proteins using the Munich Information Center for Protein Sequences functional classification system. The black bars represent the number of induced genes and the white bars represent the number of downregulated genes in each class. Because some proteins have multiple functions and may be involved in more than one biological process, the sum of the genes in each of the classifications is greater than the total number of genes. 


\section{Expression profiles}

of cell-wall-related, defense and stress, and RP genes across the spatial gradient of TuMV-GFP accumulation.

To better understand the spatial relationships between TuMV infection and the expression of host genes representative of the classes identified in the previous section, we plotted the expression levels of selected genes in mock-inoculated and TuMV-infected samples across the four zones (Fig. 4). A complete list of the 556 genes grouped according to functional class and annotated with their spatial expression patterns is provided in Supplementary Table S1. The cell wall genes exemplified by XTH6, PME3, and EXP10 all were reduced in accumulation of mRNA transcripts $(q<0.05)$. The expression of these genes was reduced most extensively in zones 0 and 1 when compared with zones 2 and 3 , whereas it was uniform across the four zones in mock-inoculated leaves (Fig. 4A). This observation demonstrates that there is a spatial relationship between viral infection and the reduced expression of this group of genes. To confirm the decreased expression of cell- wall-modification genes, the TuMV infections were repeated and zones 0 and 3 were dissected from the new foci as described for the microarray experiment. Quantitative (q)RTPCR assays were developed for XTH6 and PME3 and performed on mock-inoculated and TuMV-infected zones 0 and 3 at 5 days after inoculation (dai). As expected, the $\log 2$ ratio of the expression in the TuMV-infected sample versus the corresponding mock-inoculated sample demonstrated that both genes were downregulated in zone 0 (Fig. 5A).

Defense-related genes, HS genes, and RPs represent distinct functional groups of induced genes. $B G L 2$ is a marker gene for SA-mediated defense responses in A. thaliana and its expression is associated with systemic acquired resistance (Bowling et al. 1994). In compatible host-virus interactions, increased $B G L 2$ expression requires $\mathrm{SA}$ and additional components of defense signaling pathways, such as EDS1 (Huang et al. 2005). $B G L 2$ expression was maximal in zone 0 and near the mockinoculated baseline in zone 3 (Fig. 4B). HS genes, such as $H S P 70$, and an HS transcription factor (HSF4), also had their
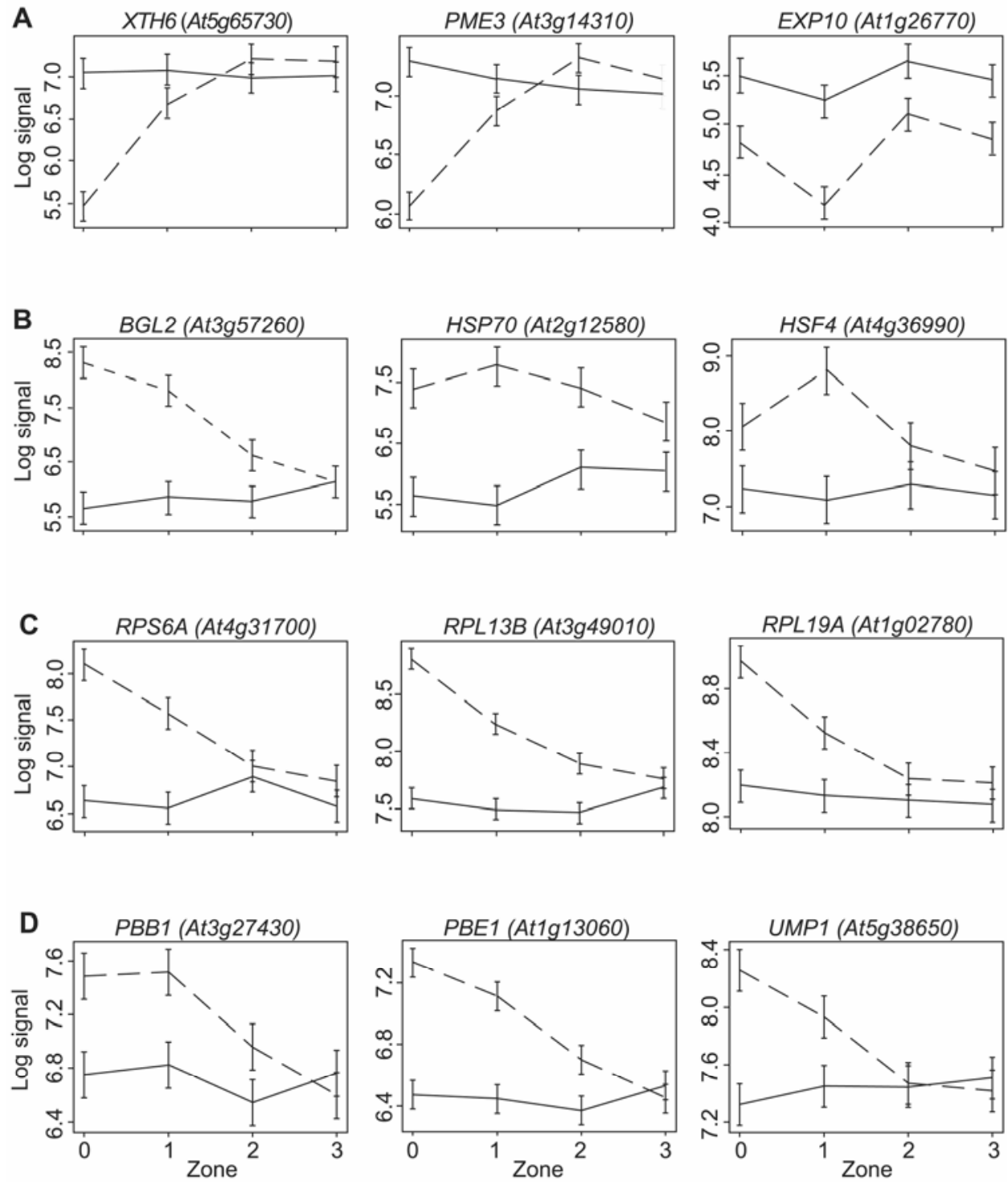

Fig. 4. Expression of selected induced and downregulated genes across the gradient of Turnip mosaic virus (TuMV) infection. The estimated least square means were plotted for TuMV-infected samples (dashed lines) and mock-inoculated samples (solid lines) for the four dissected zones to obtain spatial profiles for each gene. The plots were generated using the $\mathrm{R}$ statistical package, version 1.9.0. The error bars indicate the standard error of the mean for each least square mean ( $n$ =3). A, Genes encoding cell-wall-modification enzymes that are repressed during TuMV infection. B, Genes associated with plant defense and stress responses that are induced during TuMV infection. C, Genes encoding ribosomal proteins that are induced during TuMV infection. D, Genes encoding proteins associated with the $20 \mathrm{~S}$ core proteasome. The names of genes are provided along with their Arabidopsis Genome Initiative locus in parentheses. 
peak expression in zones 0 and 1 and possessed near-baseline expression in zone 3 (Fig. 4B). These results demonstrate that increased expression of defense-related and HS genes is dependent on the presence of the virus and is not induced in advance of the viral infection in the inoculated leaves. Thus, TuMV does not induce systemic defense responses in advance of the viral infection front. Increased expression of these defense and HS genes has been observed previously in response to TuMV (Whitham et al. 2003); therefore, we did not validate expression by qRT-PCR.

The RPs and proteasome genes were not previously identified as being induced by plant viruses. The prototypical expression profiles of TuMV-induced RPs are exemplified by RPS6A (a constituent of the 40S subunit) and RPL13B and RPL19A (constituents of the 60S subunit) (Fig. 4C). These genes were induced maximally in zone 0 , with expression decreasing to baseline in zone 3 . The expression profiles of members of the $20 \mathrm{~S}$ core proteasome $(P B B 1$ and $P B E 1)$ and $U M P 1$ are shown in Figure 4D. These data demonstrate that $\mathrm{RP}$ and proteasome genes require the presence of the virus for their induction and shared a similar expression profile with the defense-related genes. qRT-PCR assays were developed for RPS6A, PBB1, and PBE1, which independently confirmed that mRNA transcript accumulation of these genes was increased in zone 0 (Fig. $5 \mathrm{~A}$ ). Each of the RP genes shown in Figure $4 \mathrm{C}$ is a member of a multigene family. However, not every member of each family necessarily is induced by TuMV infection, indicating that there is differential regulation of expression among family members (data not shown). In the case of RPS6A and $B$, both members were induced significantly, whereas only two of the eight members of the RPL13 family were induced at our statistical threshold.

It has been suggested that plant viruses may cause a general decrease in host gene expression (Wang and Maule 1995). TuMV induced the expression of more genes than it suppressed at the 5\% FDR threshold (Fig. 3), and specific functional classes of genes were preferentially induced or downregulated over others. It is possible that the data analysis procedures might have introduced a bias against identifying downregulated genes, especially with respect to zone 0 . This bias could occur because the data were scaled to an arbitrary threshold of 500 to allow comparisons across arrays. In an experiment where the majority of genes become downregulated, this could result in an underestimation of the fold change for downregulated genes and an overestimation of the fold change for upregulated genes. The agreement of the estimated fold change ratios derived from qRT-PCR for our selected genes when compared with those derived from microarray in zone 0 suggests that there is not a systematic bias introduced by the microarray analysis (Fig. 5B). Thus, these data indicate that TuMV infection may not cause a global decrease in the accumulation of $A$. thaliana mRNA transcripts. However, it is not yet possible to draw conclusions about the global protein levels.

\section{Expression of $A$. thaliana genes}

in inoculated and systemic tissues over a 10-day time course.

The dissection studies above indicate that defense and stress responses were not induced in advance of TuMV infection in the inoculated leaves, but rather in proportion to the amount of virus accumulation. We were interested in investigating this further, because it has been reported that other viruses, such as Cauliflower mosaic virus, can induce the expression of defenserelated genes in systemic tissues ahead of the virus in compatible interactions (Love et al. 2005). To address this question, mRNA transcript abundance was assayed in parallel in inoculated and systemic rosette leaves and cauline leaves over a 10day time course. Because of the large number of samples, a custom-made microarray containing a preselected set of 388

Table 1. Sample types used for interrogating Arabidopsis thaliana responses to Turnip mosaic virus and Oilseed rape mosaic virus systemic infections

\begin{tabular}{lccccc}
\hline & \multicolumn{5}{c}{ Days after inoculation } \\
\cline { 2 - 6 } Tissue & $\mathbf{2}$ & $\mathbf{3}$ & $\mathbf{5}$ & $\mathbf{7}$ & $\mathbf{1 0}$ \\
\hline Inoculated leaf & $\mathrm{X}$ & $\mathrm{X}$ & $\mathrm{X}$ & $\mathrm{X}$ & $\mathrm{X}$ \\
Systemic rosette leaf & $\ldots$ & $\mathrm{X}$ & $\mathrm{X}$ & $\mathrm{X}$ & $\mathrm{X}$ \\
Cauline leaf & $\ldots$ & & $\mathrm{X}$ & $\mathrm{X}$ & $\mathrm{X}$ \\
Flowers & $\ldots$ & $\mathrm{X}$ & $\mathrm{X}$ & $\mathrm{X}$ & $\mathrm{X}$ \\
\hline
\end{tabular}

B

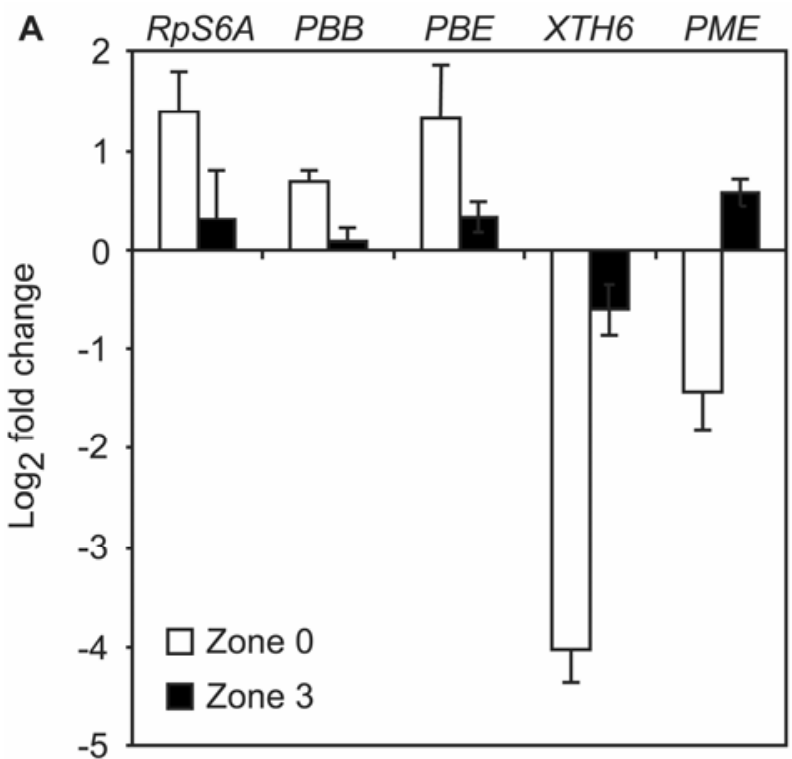

Comparison of $\log _{2}$ fold change in zone 0 for microarray and qRT-PCR

\begin{tabular}{lcc}
\hline Gene & Microarray & qRT-PCR \\
\hline RPS6A (At4g31700) & 1.5 & 1.4 \\
PBB1 (At3g27430) & 0.75 & 0.7 \\
PBE1 (At1g13060) & 0.8 & 1.3 \\
XTH6 (At5g65730) & -1.6 & -4.0 \\
PME (At3g14310) & -1.3 & -1.4 \\
\hline
\end{tabular}

Fig. 5. Analysis of the expression of selected genes by quantitative reverse-transcriptase polymerase chain reaction (qRT-PCR). RNA was extracted from zones 0 and 3 of three replicates of plants distinct from those used for microarray analyses and used in qRT-PCR reactions. A, The log 2 of the fold change is plotted here for each gene. The fold change was determined for each zone by dividing the relative expression level of each gene in the TuMV-infected sample by its relative expression level in the corresponding mock-inoculated sample. The vertical bar represents the standard error for the three replicates. B, The fold change values determined from microarray and qRT-PCR are compared. 
A
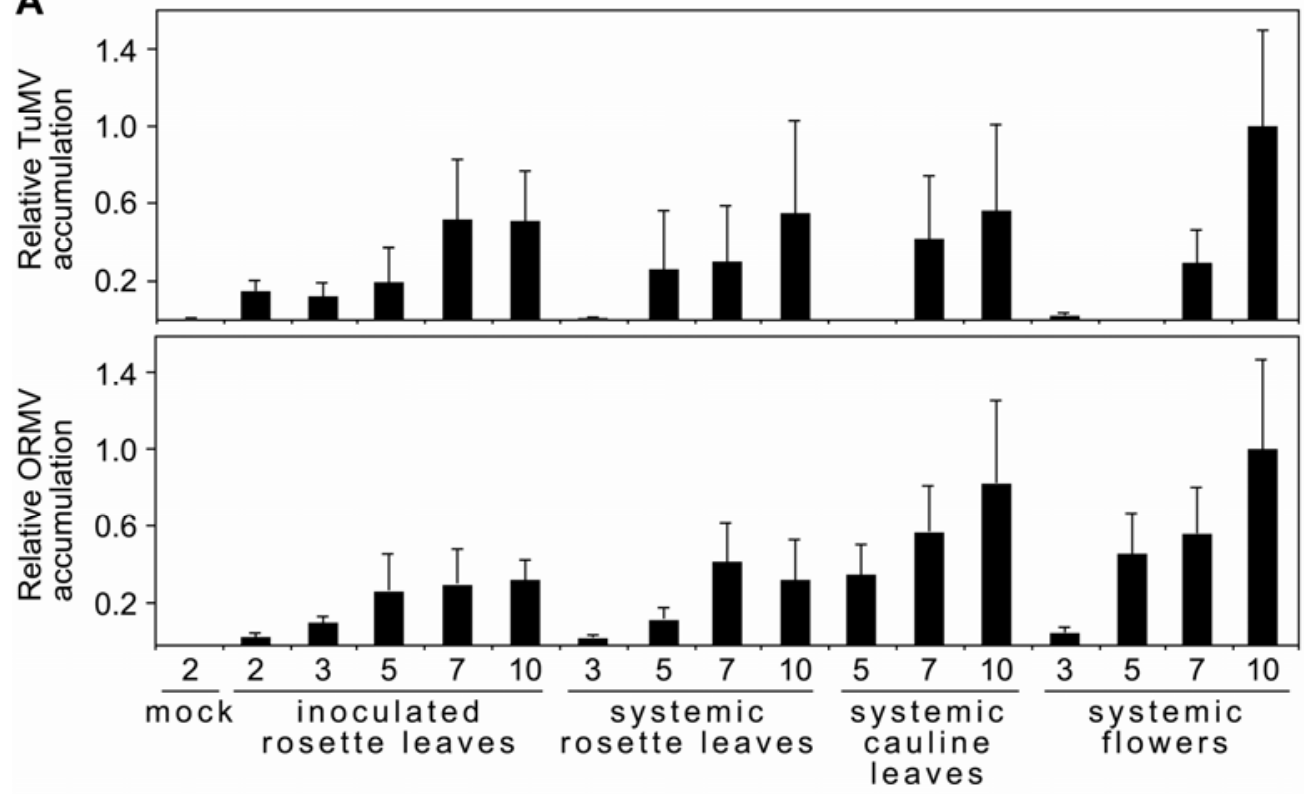

Days after inoculation

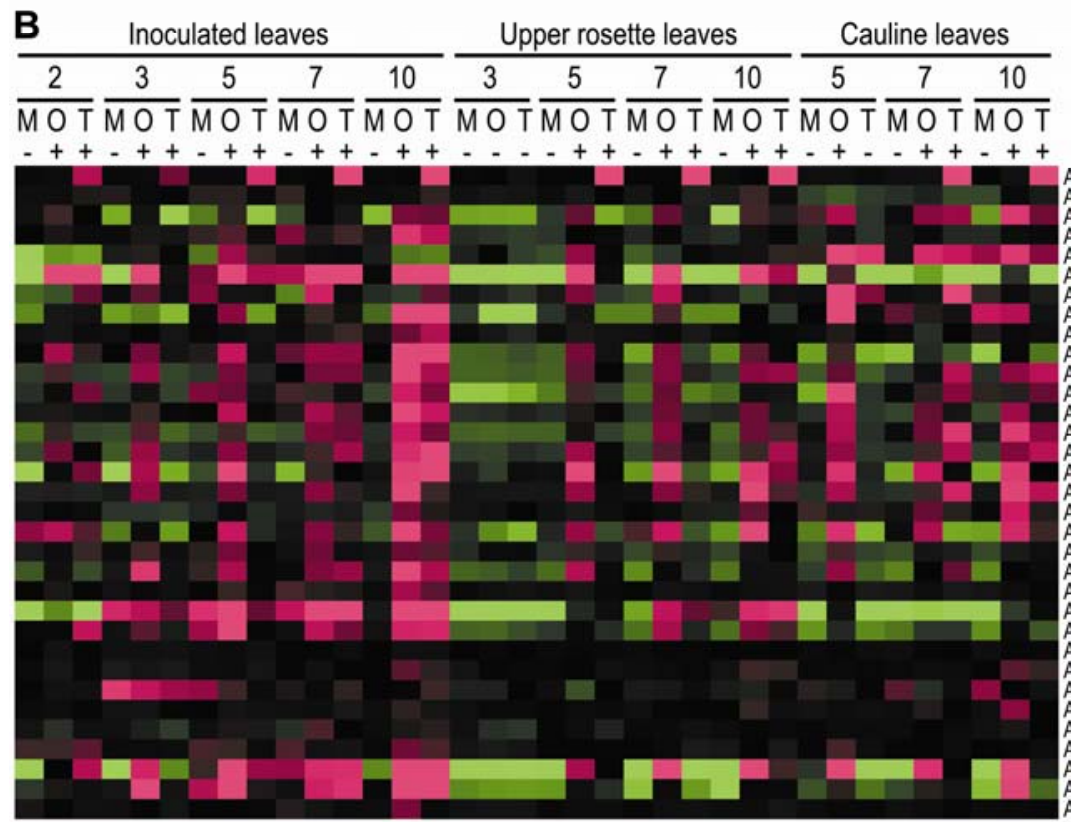
- Tissue - DAI

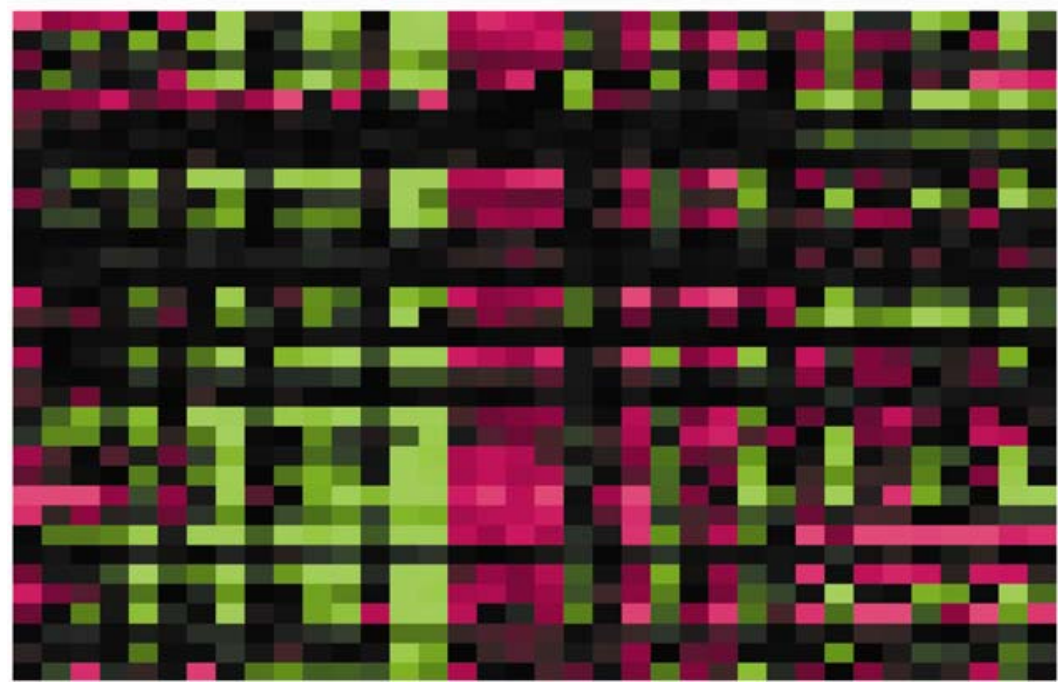

- Virus

- Accumulation

AF078810: GFP

AT3G09440: $\mathrm{HSC70-3}$

A

AT1G02920: GLUTATHIONE TRANSFERASE

AT3G48090: EDS 1 - PUTATIVE LIPASE

AT2G30550: PUTATIVE LIPASE

AT2G30140: PUTATIVE GLUCOSYLTRANSFERASE

AT4G21980: SYMBIOSIS-RELATED LIKE PROTEIN

AT2G2120: AAT1 1 :

AT5G02490: $H S C 70-2$

AT5G12030: HSP 17.6A

AT5G09590: MTHSC70-2

AT4G26060: PUTATIVE PROTEIN

AT4G39670: PUTATIVE PROTEIN

AT4G37150: HYDROXYNITRILE LYASE LIKE

AT4G30270: XYLOGLUCAN ENDOTRANSGLYCOSYLA AT2G29120: LIGAND-GATED ION CHANNEL PROTEIN

2510: PROLINE-RICH CELL WALL PROTEIN

AT1G61990: HYYOTHETICAL PROTEIN

AT 1G10410: UNKNOWN PROTEIN

AT2G14610: PR-1-LIKE

AT2G18680: UNKNOWN PROTEIN
AT1G05100: NPK1-RELATED MAP KINASE

AT4G36540: PUTATIVE PROTEIN

4G32330: PUTATIVE PROTEIN

AT4G35750: PUTATIVE E1B 19K/BCL-2-INTERACTING PROTEIN NIP2 AT1G11700: UNKNOWN PROTEIN

AT1G12100: HYBRID PROLINE-RICH PROTEIN

AT1G09750: EXPRESSED PROTIEIN AT5G01240: LAX1 IAUX1- LIKE PERMEASE 1G10290: SIMILAR TO DYNAMIN-LIKE PHRAGMOPLASTIN T1G4435: GR1PROT

T1G 27030: EXPRESSED PROTEIN

AT3G61460: BRH1, A BRASSINOSTEROID RESPONSIVE RING-H2 AT 1 G78820: GLYCOPROTEIN(EP1)

AT2G34060: PEROXIDASE

AT2G05790: SIMILAR TO ELICITOR INDUCIBLE CHITINASE AT1G76490: HMG COA REDUCTASE

AT2G27078: ELL1 1 CITIVE SERINE CARBOXYPEPTIDASE ॥

AT2G37640: SIMILAR TO ALPHA-EXPANSIN 6

ATG18970: EXPRESSED PROTEIN

AT2G26330: ERECTA

AT1G54040: JASMONATE INDUCIBLE PROTEIN

AT1G6620: BZIP DNABINDING RROTEIN AT5G43720: SBP-LIKE 
genes (Huang et al. 2005) was used to interrogate the expression of A. thaliana genes in response to TuMV and Oilseed rape mosaic virus (ORMV; genus Tobamovirus). ORMV was included in this experiment because we were interested in knowing whether the results obtained with TuMV were applicable to other viruses. In addition, ORMV induces very robust changes in A. thaliana gene expression (Huang et al. 2005, Whitham et al. 2003); thus, we expected that it might be able to cause systemic changes in host gene expression. The microarray includes many defense- and stress-related genes, and we previously used it to examine the signaling requirements for the expression of such genes in response to compatible viruses in a panel of $A$. thaliana mutants defective in defense signaling pathways (Huang et al. 2005). The identity and selection criterion for each gene on the array is provided in Supplementary Table S2.

In order to examine the expression of defense and stress genes in systemic tissues, 25 A. thaliana plants were assigned to each of three inoculation types (TuMV-GFP, ORMV, and mock), and four fully expanded rosette leaves were inoculated on each of these plants. At each of 2, 3, 5, 7, and 10 dai, samples were collected from five plants of each inoculation type and pooled to obtain the sample types indicated in Table 1. This collection process was replicated three times with different sets of plants and, in all, 144 samples (three replications $\times$ three inoculation types $\times 16$ time-tissue combinations) were obtained. RNA was extracted from the samples, labeled by the cDNA-mediated annealing, selection, extension, and ligation assay (DASL) (Fan et al. 2004) and hybridized to Sentrix array matrices representing 388 unique genes (Huang et al. 2005). Two aliquots of RNA provided a technical replication of the experiment. Background subtraction was performed after scanning and then an average signal was computed for each gene based on the signals from three unique probes for each gene. As shown by Tusher and associates (2001), the data from both technical replicates were cube-root transformed to achieve approximate normality and constant variance for each gene on the array. The transformed data from technical replications were averaged prior to statistical analysis to produce 388 measures of expression for each RNA sample. A gene-specific linear mixed model analysis was performed separately for each tissue to determine genes that were altered significantly in their expression profiles in response to TuMV or ORMV compared with the corresponding mock-inoculated treatment (discussed below). Subsequently, a $q$ value was calculated for each gene to estimate the FDR (Benjamini and Hochberg 1995; Manly et al. 2004; Storey and Tibshirani 2003). The FDR ( $q$ value) and $P$ value obtained for each gene for each combination of tissue and virus is provided in Supplementary Table S3. These analyses provided 67 genes with expression profiles that were altered in response to TuMV with an estimated FDR of $5 \%(q<0.05)$. Nearly all of these also were altered in ORMV infections, indicating a common response to the two viruses, as has been described previously (Whitham et al. 2003).

In order to correlate virus accumulation with host gene expression in each tissue, the relative accumulation of TuMVGFP in each sample type was determined from the signal of $g f p$-specific (AF078810) oligos present on the microarray, and ORMV accumulation was determined by RNA gel blot (Fig. 6A). These data confirmed that TuMV and ORMV progressively spread throughout the inoculated plants. The accumulation of each virus was highest in the systemic flower tissues at 10 dai and, thus, levels of TuMV and ORMV were plotted relative to this tissue and timepoint.

To understand the relationships of the expression profiles among the 67 most significant TuMV-responsive genes identified from the comparison of the mock-inoculated tissue to the corresponding TuMV-infected tissue, $\mathrm{K}$-means clustering was used to place these genes into one of two groups with related expression profiles (Fig. 6B) (Eisen et al. 1998). To ensure effective clustering, the data first were median centered to standardize the expression of each gene across samples. As seen in Figure 6B, expression values above the median were assigned the magenta coloring, black was near the median, and green was below the median. For each gene, the signal intensity in mock-inoculated and virus-infected samples can be compared independently for each tissue and timepoint. A key to interpreting this figure is that it is necessary to compare the relative expression of a given gene across sample types and not necessarily the colors that are used to represent the expression level. Figure 6B illustrates how the presence of each virus is positively correlated with altered host gene expression profiles, and that these responses to TuMV and ORMV are similar across the different leaf types. Because only 2 of the 67 genes were significantly altered in flowers at the 5\% FDR, flower samples were excluded from this analysis.

Genes in the top cluster of Figure 6B were induced primarily in response to TuMV and ORMV in the different leaf types, and their increased expression was synchronous with the kinetics of virus accumulation. Members of this cluster include defense-related genes such as $P R-1$, glutathione transferase (At1g02920), and EDS1. This cluster of genes also contains several HS or HS-related genes, such as BIP-2, HSP17.6A, HSC70-2, HSC70-3, HSP70, and a putative HS transcription factor (At2g26150). Induction of HSP17.6A in response to TuMV was significant in flowers $(q<0.05)$, and examination of the expression profiles of other HS-related genes, including HSP70 and the HS transcription factor, were as well (data not shown). Although their $q$ values were $>0.05$, these genes are detected consistently as induced in a variety of tissues in response to TuMV and ORMV over this time course. The quantification of each virus showed that there was high variation in their accumulation across the three replicates (Fig. 6A), which

Fig. 6. Induction or downregulation of genes in inoculated and systemic leaf tissues. A, Relative accumulation of Turnip mosaic virus (TuMV) and Oilseed rape mosaic virus (ORMV) in inoculated and systemic leaves and flowers over a 10-day time course. Because the TuMV genomic RNA is polyadenylated, TuMV-green fluorescent protein (GFP) was quantified directly by the microarray probes for GFP (AF078810). The genomic RNA of ORMV was detected with a coat protein probe by Northern blot hybridization for each of the three replicates. The signal of the genomic RNA band was quantified by phosphorimager and then it was normalized to the signal from 18S rRNA. The accumulation of TuMV and ORMV in each sample type was plotted relative to the 10-day after inoculation (dai) flower samples which accumulated the highest amounts of each virus. The error bars indicate the standard error for the signals from each of the three replicates. B, Expression of host genes in inoculated leaves and systemic leaves over a 10-day infection time course. These 67 host genes have expression profiles altered significantly $(q<0.05)$ in response to TuMV in one or more tissues. The expression values for each gene were median centered and grouped into one of two clusters using K-means clustering. The results were visualized with the TreeView program (Eisen et al. 1998), Each colored box represents the average signal of a gene determined from three biological and two technical replicates in the indicated sample. Magenta indicates samples in which the gene was expressed above the median for the 36 sample types, black represents samples in which expression was at or near the median, and green represents expression below the median. The intensity of color corresponds to the degree to which expression was above or below the median. M, mock inoculated; T, TuMV-GFP infected; O, ORMV infected; (+) or (-) indicates whether virus was detected above background for each timepoint and treatment. 
likely contributes to variability in host gene expression. This also may be a significant factor in the inability to identify significant genes in the flower samples. The lower cluster contains genes that are primarily downregulated in response to TuMV and ORMV infection, but they were not considered to be downregulated in response to either virus in flowers at the $q$ value cut off of 0.05 . In contrast to the upregulated genes, the expression of these genes decreased to a greater extent as TuMV and ORMV accumulated in inoculated or systemic leaves. These downregulated genes include expansins, which may be involved in cell wall extensibility and could contribute to the stunted-growth phenotype of TuMV-infected plants. For both the induced and downregulated genes, it is apparent that their expression is highly dependent on the presence and accumulation of TuMV or ORMV (Fig. 6B). These data suggest that these two viruses do not induce defense and stress genes or downregulate genes in advance of the infection front; rather, most host gene expression changes are directly dependent on virus accumulation in leaf tissues. It is significant to note here that the induction or downregulation of genes observed in Figure $6 \mathrm{~B}$ is consistent with our previous data (Whitham et al. 2003) with two exceptions, At1g19050 and At1g44350. These two genes may be false discoveries in both data sets or they may not respond reliably in TuMV infections.

\section{DISCUSSION}

\section{TuMV infection modulates $A$. thaliana mRNA transcript in proportion to its accumulation.}

We coupled a macrodissection approach with DNA oligonucleotide microarray profiling of mRNA transcript abundance to gain insight into the spatial and temporal relationships between viral infection and host gene expression with a focus on TuMV, a potyvirus that causes severe symptoms in the Col- 0 ecotype of A. thaliana. We defined four zones based on the average size of infection foci at 5 dai and the diameter of the

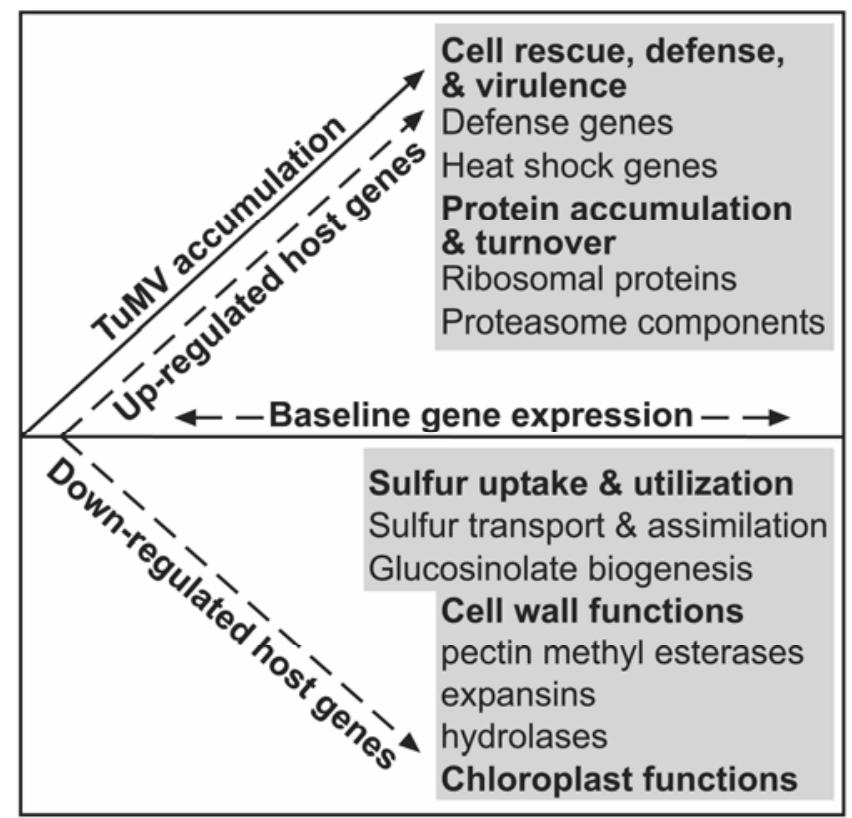

Time after inoculation $\rightarrow$

Fig. 7. Relationship of Turnip mosaic virus (TuMV) accumulation to the expression of major functional groups of genes. Gene functional classes are depicted in bold text in gray boxes and subcategories of genes within each functional class are depicted in plain text. The solid arrow represents virus accumulation and the dashed arrow represents the up- or downregulation of the indicated gene functional groups. micropunch used for dissection. TuMV accumulation was present in a decreasing gradient radiating from zone 0 out to 2 , with no evidence for replication or GFP accumulation yet in zone 3. Analysis of the expression profiles of genes for which mRNA transcript abundance was significantly altered enabled us to draw conclusions about when the expression of different functional groups of genes became altered with respect to the location of the virus in leaf tissues. Based on interesting functional groups of genes and their mRNA expression profiles, we propose a general model that describes the relationships between virus accumulation and modulation of host gene expression (Fig. 7). Examples of upregulated genes include those involved in basal host defense and stress responses and genes associated with protein synthesis and turnover that have the potential to act as host factors. Examples of downregulated genes are those associated with chloroplast functions, sulfate utilization, or cell wall expansion. Decreased expression of these genes is expected to lead to physiological changes consistent with disease symptoms. Regardless of whether the genes were up- or downregulated, changes in mRNA transcript abundance were dependent on the amount of virus accumulation in a given cell or tissue and not on time after inoculation.

The brief exposure to UV light was of potential concern in the dissection experiment, because it alone can cause significant and predictable changes in gene expression (Ulm and Nagy 2005). We compared the 556 TuMV-responsive genes identified at the 5\% FDR with those identified following exposure of $A$. thaliana to different wavelengths of light in the UV$B$ range (Ulm et al. 2004). There was no evidence that the altered transcript accumulation was caused by UV light, because there was very little overlap of genes between the virus and UV light experiments. Combined with the fact that the virusresponsive genes were expressed at nearly constant levels across the four zones in the mock-inoculated control plants, we concluded that the brief exposure to UV light did not contribute to the differential expression of genes observed between the mock-inoculated and TuMV-infected plants.

The spatial analyses confirmed effects on the host transcriptome that have been reported previously (Golem and Culver 2003; Itaya et al. 2002; Senthil et al. 2005; Whitham et al. 2003), providing further support that the experimental approaches were sound. One major functional class of genes that was induced by viral infection is associated with cell rescue, defense, and virulence. Previous studies of infected A. thaliana and tomato leaves have revealed that as many as one-third of the genes commonly induced by viruses are known or predicted to be involved in plant defense and stress responses (Whitham 2004). However, in the dissection study, only approximately $1 / 10$ of the induced genes were in the defense and stress category. This discrepancy probably is due to the more effective sampling strategy that allowed additional genes to be identified other than those that most dramatically change in expression level. We found that the expression of defense and stress-related genes, such as PR and HS genes, did not occur in advance of the virus infection in whole plants or across spatial gradients in inoculated leaves (Figs. 3B and 6B). Interestingly, these types of genes were induced most strongly later, in cells or tissues where virus accumulation was most abundant. In compatible host-virus interactions, an SA-dependent pathway controls expression of a cohort of defense-related genes (Huang et al. 2005), but it has no effect on HS genes (Carr et al. 2006). The data presented here demonstrate that SA-dependent defense-like responses are not induced systemically ahead of TuMV or ORMV infection in these compatible interactions.

Current models for the spatial effects of viral infection on host gene expression are based on in situ hybridization of selected host genes in virus-infected cotyledons. These studies have led 
to the proposal that major changes occur at an early stage of infection, in the infected cells immediately adjacent to healthy cells (Maule et al. 2002). Our results indicate that most changes in mRNA transcript abundance occur most strongly later in TuMV infection and not necessarily in virus-infected cells that are immediately adjacent to healthy cells. We have obtained similar results from a micropunch experiment with Soybean mosaic virus tagged with GFP in soybean (C. Yang and S. A. Whitham, unpublished). It appears that some time is required before many significant changes begin to happen, indicating that there is a threshold of viral RNA and proteins required. Another contributing factor is that there may be a lag time for the affected mRNA transcripts to increase or turn over sufficiently to be detected by these gene expression assays and the statistical analysis. Considering the differences in host-virus systems, plant tissues, and approaches to determine the genes expression changes, our study may not be in conflict with previous reports. In pea cotyledons infected with Pea seed born mosaic virus, host gene expression is modulated in a narrow band of cells in which virus replication is most active. For the TuMV-A. thaliana interaction, there apparently is not a narrow band of cells supporting replication as the virus spreads throughout infected leaf tissue. In contrast, TuMV accumulation is sustained as foci continue to expand, at least through 5 dai. This TuMV gradient that expands radially out from the center of foci is distinct from the transient wave of replication observed in cotyledons. As shown in cotyledons and here in A. thaliana leaves, most gene expression changes occur in cells that support the greatest amount of virus accumulation. It will be interesting to investigate these gene expression changes further with viral mutants that do not accumulate to the same levels as the wild type or in mutants that accumulate to similar levels in the host but do not cause symptoms.

\section{A large proportion of newly identified, induced genes are involved in protein synthesis and turnover.}

We reasoned that many genes with virus-altered expression profiles were not identified previously because RNA samples used for expression analyses were derived from whole leaves infected with viruses (Golem and Culver 2003; Itaya et al. 2002; Senthil et al. 2005; Whitham et al. 2003). The use of whole leaves was expected to mask interesting gene expression changes or dilute them, so that they could not be detected. Thus, we expected to identify gene expression changes that had not been observed before using the macrodissection strategy. A very striking finding was the degree to which suites of genes encoding proteins involved in protein synthesis were induced in response to TuMV (Fig. 3B). The protein products of 69 genes in the protein synthesis category are known or predicted to be constituents of either the $60 \mathrm{~S}$ or $40 \mathrm{~S}$ ribosomal subunits. There are approximately 78 different RPs in eukaryotes that assemble with the four ribosomal RNAs to form the ribosome (Planta and Mager 1998; Venema and Tollervey 1999). RP genes, such as RPS6A, RPL13B, and RPL19A, had the highest expression in zone 0 and baseline expression in zone 3 . Similar to the defense genes, RP expression was strongly induced in cells containing the highest levels of virus accumulation. Interestingly, silencing of RPs by RNA interference has demonstrated that they play an important role in the replication of Drosophila $\mathrm{C}$ virus (DCV) and poliovirus (Cherry et al. 2005). RNAi silencing of 66 Drosophila ribosomal genes, including Rps6 and Rpl19, had a specific, deleterious effect on DCV replication, which depends on an internal ribosome entry site (IRES) to initiate translation. The replication of vesicular stomatitis virus, which does not possess an IRES, was not affected in Drosophila cells when RPs were silenced. It will be interesting to test whether TuMV, which uses cap-independent translation that might be mediated by an IRES (Gallie 2001; Zeenko and Gallie 2005), utilizes induced plant RPs.

The increased expression of so many RPs in response to TuMV suggests that their expression is coordinately regulated. The response may be due to the plant's need to compensate for the loss in ability to synthesize its own proteins or, perhaps, TuMV is able to induce the expression of these genes in order to increase the capacity of the cell to synthesize proteins. In Drosophila melanogaster, the expression of RPs appears to be under a type of control that regulates their stoichiometry. For example, RNAi silencing of Rpl19 and other RPs that affected DCV replication caused a concomitant decrease in Rps6, which shares no sequence similarity (Cherry et al. 2005). In yeast, the expression of RPs in response to a nonfermentable carbon source or nitrogen is highly coordinated and has been associated with specific regulatory elements in the promoter regions (Goncalves et al. 1995; Mager and Planta 1991). Similar mechanisms may function in plant cells and could account, in part, for the highly coordinated induction of these proteins in response to TuMV.

\section{Groups of downregulated genes have functions consistent} with those expected to be involved in symptom development.

Previous host transcriptome analyses have focused more on induced genes than downregulated genes and have highlighted defense- or stress-like responses. The spatial sampling strategy and accompanying statistical analyses allowed us to identify significantly downregulated host genes. As with the induced genes, the downregulated genes were identified primarily in zones 0 and 1, where the most virus accumulation had occurred. The groups of genes that are downregulated have biases in favor of different functions or cellular locations when compared with the induced genes. For example, the majority of genes with known or predicted functions in the chloroplast are downregulated, as are those with a function in the cell wall (Fig. 3A). A survey of the MIPS functional classes shows that there clearly are classes in which downregulated genes are either absent or underrepresented (Fig. 3B). Thus, there probably is not a general decrease in mRNA expression, but a more selective reason why the expression of certain groups of genes becomes repressed. As more proteomics studies become available for plant viruses, it will be possible to determine whether this conclusion holds true at the protein level. The reduced expression of sets of chloroplast, sulfate assimilation, and cell wall extensibility genes suggests possible mechanisms for the appearance of chlorosis and stunted growth in the TuMVinfected A. thaliana plants. Symptoms of Potyvirus-infected plants are associated with the activities of HC-Pro, which is a suppressor of RNA silencing that interferes with miRNA function and causes developmental defects. The altered regulation of many of the genes we have identified eventually may be linked to misregulation of the expression of certain miRNAregulated transcription factors due to TuMV infection. Efforts to identify such transcription factors in our dataset were not successful, suggesting that this approach was not suitable for identifying miRNA-regulated genes even though considerable evidence has demonstrated that HC-Pro does, indeed, alter the expression of such genes (Chapman et al. 2004; Kasschau et al. 2003; Mallory et al. 2002).

\section{Potential applications of this dissection strategy in other host-pathogen interactions.}

The dissection strategy employed here was simple and should be generally applicable to any plant virus for which an infectious clone expressing GFP or other marker genes that allows for nondestructive visualization of infection foci is available. The new types of co-regulated genes that were iden- 
tified as a result reveal new insight into ways in which viruses such as TuMV alter the biology of their hosts. Other kinds of plant-pathogen interactions also involve localized interactions between microbes and cells of their hosts; thus, we expect that this dissection strategy will be effective for studying events associated with the hypersensitive response or compatible interactions between plants and bacterial or fungal pathogens. Although localized interactions between a particular host and pathogen can take on different forms, the cells of a given tissue (e.g., leaf) comprise a gradient of stages of infection ranging from late to early to noninfected. Defining how hosts respond to infection across these spatial gradients will be critical to understanding mechanisms of pathogenesis as well as how plant defenses are deployed in resistance.

\section{MATERIALS AND METHODS}

\section{Dissection of TuMV-GFP infection foci.}

To prepare TuMV-GFP inoculum, tungsten particles were coated with p35TuMVGFP plasmid (Lellis et al. 2002) and bombarded into Nicotiana benthamiana leaves (Gal-On et al. 1997). Sap from $N$. benthamiana leaves was used to inoculate turnip plants ('Top crop') to further amplify the virus. TuMVGFP-infected turnip leaves were ground in $20 \mathrm{mM}$ sodium phosphate buffer ( $\mathrm{pH} 7.2,1: 6, \mathrm{wt} / \mathrm{vol})$, filtered through Miracloth (Calbiochem, San Diego, CA, U.S.A.), and frozen in aliquots at $-80^{\circ} \mathrm{C}$. The titer of the inoculum was adjusted to yield well-separated GFP foci.

Col-0 plants were grown for 7 weeks in $10 \mathrm{~h}$ of light at $22^{\circ} \mathrm{C}$ to allow large rosette leaves to develop. Seven to eight rosette leaves from each of five plants were dusted with Carborundum and rub inoculated with TuMV-GFP using a cotton-stick applicator. A corresponding set of five control plants was dusted with Carborundum and mock inoculated with a cotton-stick applicator that was soaked in uninfected turnip sap diluted in phosphate buffer. At 120 hai, a 1.2-mm Harris micropunch (Whatman, Florham Park, NJ, U.S.A.) was used to dissect three to seven foci per infected or mock-inoculated leaf into four zones under UV illumination (100-W Blak-Ray longwave UV lamp; UVP, Upland, CA, U.S.A.). The area of the punch was equivalent to approximately 770 epidermal cells and each punch was 26 to 30 cells across. Leaves were under UV illumination for a maximum of $3 \mathrm{~min}$. Leaf punches were placed into 1.5-ml tubes filled with RNAlater solution (Ambion, Austin TX, U.S.A.) and stored at $-20^{\circ} \mathrm{C}$. All the leaf punches from the same treatment and zone were pooled together to yield 8 total samples and a grand total of 24 samples for the three independent biological replicates.

\section{RNA extraction, amplification, labeling, and generation of GeneChip MAS 5.0 data.}

Total RNA was extracted using RNAqueous (Ambion) and the concentration and quality of each sample were determined by a NanoDrop spectrophotometer (NanoDrop Technologies, Wilmington, DE, U.S.A.) and by RNA Nanochip on a 2100 Bioanalyzer (Agilent Technologies, Inc., Palo Alto, CA, U.S.A.). The RNA samples (50 ng) then were amplified and labeled with the Ovation Biotin RNA Amplification and Labeling System (NuGEN Technologies, Inc. San Carlos, CA, U.S.A.) (Dafforn et al. 2004). Each amplified and labeled RNA sample was hybridized to an Arabidopsis ATH1 genome array (Affymetrix, Inc.) containing more than 22,500 probe sets representing approximately 24,000 genes (Redman et al. 2004). The RNA samples were processed in a random order to avoid systematic biases that can be introduced by the position in the hybridization and wash queue. Signal intensities of scanned microarrays were generated with Affymetrix MAS 5.0 suite.
Global scaling was applied to allow comparison of gene signals across multiple microarrays and a scaling factor was applied for each chip to adjust the average signal of the microarray to an arbitrary target of 500 .

\section{Statistical analysis \\ to identify differentially expressed host genes.}

To identify genes whose zonal expression profiles were affected by TuMV-GFP infection, gene-specific mixed linear model analyses of the log-normalized signal data were conducted using the SAS mixed procedure (Chu et al. 2002; Wolfinger et al. 2001). For each gene, the mixed linear model included fixed effects for treatment (mock or TuMV-GFP infection), zone $(0,1,2$, or 3$)$, and treatment-zone interaction, along with random effects for replication, treatment-replication, and residual error terms. $P$ values from SAS results were converted to $q$ values (Storey and Tibshirani 2003) using the $\mathrm{R}$ program (version 1.9.0). These $q$ values were used to produce lists of differentially expressed genes as described for the analysis of the fiber-optic bead array data. Estimates of the mean expression level for each combination of treatment and zone were produced from the mixed linear model analyses and plotted to show the expression levels of selected genes in mock-inoculated and TuMV-infected samples across the four zones.

\section{RT-PCR analysis of TuMV RNA in infection foci.}

Reverse-transcription reactions contained $400 \mathrm{ng}$ of DNaseItreated RNA from each of the four zones dissected from mockinoculated or TuMV-infected leaves. The reactions were primed with either oligo(dT) to amplify the $(+)$ strand of TuMV or TuMVCPL to amplify the (-) strand, and Superscript II reverse transcriptase (Invitrogen, Carlsbad, CA, U.S.A.) was used to synthesize first-strand cDNA. PCR amplification of TuMVCP was performed with the TuMVCPL and TuMVCPR primers for 25 cycles at $95^{\circ} \mathrm{C}$ for $30 \mathrm{~s}, 55^{\circ} \mathrm{C}$ for $30 \mathrm{~s}$, and $72^{\circ} \mathrm{C}$ for $30 \mathrm{~s}$. Amplification conditions were similar for ACTIN8, except $58^{\circ} \mathrm{C}$ was used as the annealing temperature. The sequences of these primers were TuMVCPL, 5'-TGGCTGATTA CGAACTGACG-3'; TuMVCPR, 5'-CCTCTCCGTGTTCTCT ACCG-3'; Actin8RTL, 5'-GAGACATCGTTTCCATGACG-3'; and Actin8RTR, 5'-TCCGAGTTTGAAGAGGCTACA-3'.

\section{qRT-PCR analysis.}

New RNA samples corresponding to zones 0 and 3 were collected from three biological replicates for validation of gene expression by qRT-PCR as described above for microarray analyses. Reverse-transcription reactions contained $400 \mathrm{ng}$ of DNaseI-treated RNA, oligo(dT) primer, and Supercript II reverse transcriptase (Invitrogen). qRT-PCR assays were performed using the SYBR Green Supermix kit (Bio-Rad, Hercules, CA, U.S.A.) on an iQ Real-Time PCR Detection System

Table 2. Sequence and annealing temperature (Temp.) of primer pairs used for quantitative reverse-transcriptase polymerase chain reaction

\begin{tabular}{llc}
\hline Primer & \multicolumn{1}{c}{ Sequence $\left(\mathbf{5}^{\prime}\right.$ to $\left.\mathbf{3}^{\prime}\right)$} & Temp. $\left({ }^{\mathbf{o}} \mathbf{C}\right)$ \\
\hline actin8qF & CCATGACGGGATCACATTTC & 61 \\
actin8qR & CAAACGCTGTAACCGGAAAG & 61 \\
AT4G31700LPA & GTTCTCTTTTCTTCTCCGGCTA & 59 \\
AT4G31700RPA & CATGAAACAAGCCCAAAGC & 59 \\
At5g65730XTHL & CCTCATAATTGGTGGGAAGG & 59 \\
At5g65730XTHR & GTGGTGGGACAGGAAACCTA & 59 \\
At3g27430PBB1L & GTTGGTGAAGCTATGGAGGAA & 64 \\
At3g27430PBB1R & GGAAGAAGACAAGACTGGGAAG & 64 \\
At3g14310PMEL & TGAAGCAAATCGTGTTGTCC & 59 \\
At3g14310PMER & CCCGCATTATTTCATCTCGT & 59 \\
At1g13060PBE1L & GGGAGACAGGTTTTCAGTCG & 61 \\
At1g13060PBE1R & CTCCTTGCTAACTCGGAAGC & 61 \\
\hline
\end{tabular}


(Bio-Rad). Each reaction contained $12.5 \mu \mathrm{l}$ (reaction mix), 5 pmol of each forward and reverse primer (Table 2), $1.0 \mu \mathrm{l}$ of cDNA template (previously diluted 1:40), and water to $25 \mu \mathrm{l}$. Samples were incubated for $10 \mathrm{~min}$ at $50^{\circ} \mathrm{C}$, then $5 \mathrm{~min}$ at $95^{\circ} \mathrm{C}$, followed by 45 cycles of $95^{\circ} \mathrm{C}$ for $15 \mathrm{~s}$ and $30 \mathrm{~s}$ at the appropriate annealing temperature (Table 2). Relative quantification was performed using the standard curve method, and transcript accumulation of each gene was normalized to the quantity of ACTIN 8, which does not respond to viral infection. The fold change for each zone was calculated by dividing the relative expression level of the TuMV-infected sample by the corresponding mock-inoculated sample. These values were transformed by the log of base 2 and plotted in Figure 5 .

\section{Measurement of $A$. thaliana gene expression in various tissues.}

Col-0 A. thaliana plants were grown to 21 days of age at $22^{\circ} \mathrm{C}$ with a 14-h photoperiod. The plants were assigned to three treatments: mock inoculation or inoculation with either TuMV-GFP or ORMV. Four fully expanded leaves on each of 25 plants were labeled with a Sharpie marker and dusted with Carborundum (320 grit; Fisher Scientific, Hampton, NH, U.S.A.). Virions were diluted to $0.1 \mathrm{mg} / \mathrm{ml}$ in $20 \mathrm{mM}$ potassium phosphate buffer ( $\mathrm{pH} 7.2)$ and $10 \mu \mathrm{l}$ of solution was applied to each leaf for rub inoculation. Mock-inoculated plants were treated in the same way except with phosphate buffer alone. At 2, 3, 5, 7, and 10 dai, samples were collected from 20 leaves (4 leaves from each of five plants) per treatment from either inoculated or uninoculated sites according to Table 1. The layout of this experiment was consistent with a split-splitplot design, with replicates as blocks, day as the whole-plot factor, virus as the split-plot factor, and tissue as the split-splitplot factor. In each of the three replicates of this experiment, each pool of 20 inoculated leaves, systemic rosette leaves, and cauline leaves was considered to be one split-split-plot experimental unit. The three replicates were performed in parallel in different locations within a growth room by different individuals. Total RNA was extracted from each sample type using the RNAwiz reagent (Ambion), diluted to $100 \mathrm{ng} / \mu \mathrm{l}$, and divided into two identical aliquots for independent microarray analysis. RNA samples were shipped on dry ice to Illumina, Inc. (San Diego, CA, U.S.A.), where they were labeled by the DASL assay and transcript levels were measured for each of 388 genes on the virus-host fiberoptic bead array. Detailed procedures for labeling, hybridization, scanning, image processing, and data extraction software were described previously for this and other Sentrix arrays (Fan et al. 2004; Huang et al. 2005). Briefly, each bead type (corresponding to one specific probe sequence) is represented by an average of 30 beads on the array. Bead signals are computed with weighted averages of pixel intensities, and local background is subtracted. Signal for each bead type is calculated by averaging corresponding bead signals with outliers removed (using median absolute deviation) (Fan et al. 2004; Galinsky 2003). An average signal was computed for each gene based on the signals from the three unique probes representing each gene. Pearson correlation coefficients obtained for the background-subtracted data from the two replicates of each sample typically were between 0.97 and 1.00 , demonstrating the very high reproducibility of the assay.

\section{Statistical analysis of fiberoptic bead array data.}

The background-corrected data were cube-root transformed to obtain approximate normality and within-gene constant variance for the residual random effects. As shown by Tusher and associates (2001), we used the cube-root transformation because the natural log transformation (which typically works well for cDNA and oligonucleotide array data) failed to stabi- lize the variance for the fiberoptic bead array data. The transformed data from technical replications were averaged prior to statistical analysis to produce 388 measures of expression for each RNA sample.

Modeling all tissue types together was ineffective due to heterogeneity of variance across tissue types within gene. Thus, two separate mixed model analyses were conducted for each gene-one for inoculated and systemic rosette leaves and one for cauline leaves. The mixed linear model for inoculated and systemic rosette leaves had a separate mean for each combination of inoculation type, day, and leaf type (inoculated versus systemic). Random effects for replication, replication-day interaction, and replication-day-inoculation type interaction were included to account for potential correlations among observations caused by our data collection process. Mixed models for the cauline leaves was simpler because they involved only one tissue type. Fixed factors included inoculation type, day, and inoculation-day interaction. Random factors included replication and replication-day interaction. In all analyses, tests of interest involved a comparison of the expression profile within a particular tissue under viral inoculation with the expression profile within that same tissue under mock inoculation. For each test, the SAS mixed procedure was used to obtain one $P$ value for each gene. These $P$ values were converted to $q$ values (Storey and Tibshirani 2003) using the R program (version 1.9.0). These $q$ values were used to produce lists of genes with approximate FDR of $5 \%$ by including all genes with $q$ values $<0.05$.

\section{ACKNOWLEDGMENTS}

This work was supported by the United States Department of Agriculture-National Research Initiative (02-35319-12566), Hatch Act and State of Iowa Funds, and the Iowa State University Plant Sciences Institute. We thank J. Dittman for assistance with plant growth, Z. Huang for assistance with virus inoculation and preparation of RNA samples for fiberoptic bead array assay, and A. Lellis and J. Carrington for TuMV-GFP. We thank A. Miller and M. van de Mortel for critically reading the manuscript and for helpful discussions.

\section{LITERATURE CITED}

Aparicio, F., Thomas, C. L., Lederer, C., Niu, Y., Wang, D., and Maule, A J. 2005. Virus induction of heat shock protein 70 reflects a general response to protein accumulation in the plant cytosol. Plant Physiol. 138:529-536.

Aranda, M. A., Escaler, M., Wang, D., and Maule, A. J. 1996. Induction of HSP70 and polyubiquitin expression associated with plant virus replication. Proc. Natl. Acad. Sci. U.S.A. 93:15289-15293.

Benjamini, Y., and Hochberg, Y. 1995. Controlling false discovery rate: A practical and powerful approach to multiple testing. J. R. Stat. Soc. B. 57:289-300.

Berardini, T. Z., Mundodi, S., Reiser, L., Huala, E., Garcia-Hernandez, M., Zhang, P., Mueller, L. A., Yoon, J., Doyle, A., Lander, G., Moseyko, N., Yoo, D., Xu, I., Zoeckler, B., Montoya, M., Miller, N., Weems, D., and Rhee, S. Y. 2004. Functional annotation of the Arabidopsis genome using controlled vocabularies. Plant Physiol. 135:745-755.

Bowling, S. A., Guo, A., Cao, H., Gordon, A. S., Klessig, D. F., and Dong, X. 1994. A mutation in Arabidopsis that leads to constitutive expression of systemic acquired resistance. Plant Cell 6:1845-1857.

Carr, T., Wang, Y., Huang, Z., Yeakley, J. M., Fan, J. B., and Whitham, S. A. 2006. Tobamovirus infection is independent of HSP101 mRNA induction and protein expression. Virus Res. 121:33-41.

Carrington, J. C., and Whitham, S. A. 1998. Viral invasion and host defense: Strategies and counter-strategies. Curr. Opin. Plant Biol. 1:336-341.

Chapman, E. J., Prokhnevsky, A. I., Gopinath, K., Dolja, V. V., and Carrington, J. C. 2004. Viral RNA silencing suppressors inhibit the microRNA pathway at an intermediate step. Genes Dev. 18:1179-1186.

Chen, J., Li, W. X., Xie, D., Peng, J. R., and Ding, S. W. 2004. Viral virulence protein suppresses RNA silencing-mediated defense but upregulates the role of microRNA in host gene expression. Plant Cell 16:1302-1313.

Cherry, S., Doukas, T., Armknecht, S., Whelan, S., Wang, H., Sarnow, P., and Perrimon, N. 2005. Genome-wide RNAi screen reveals a specific sensitivity of IRES-containing RNA viruses to host translation inhibi- 
tion. Genes Dev. 19:445-452.

Chu, T., Weir, B., and Wolfinger, R. D. 2002. A systematic statistical linear modeling approach to oligonucleotide array experiments. Math. Biosci. 176:35-51.

Dafforn, A., Chen, P., Deng, G., Herrler, M., Iglehart, D., Koritala, S., Lato, S., Pillarisetty, S., Purohit, R., Wang, M., Wang, S., and Kurn, N. 2004. Linear mRNA amplification from as little as $5 \mathrm{ng}$ total RNA for global gene expression analysis. Biotechniques 37:854-857.

Dunoyer, P., Lecellier, C. H., Parizotto, E. A., Himber, C., and Voinnet, O. 2004. Probing the microRNA and small interfering RNA pathways with virus-encoded suppressors of RNA silencing. Plant Cell 16:1235-1250.

Eisen, M. B., Spellman, P. T., Brown, P. O., and Botstein, D. 1998. Cluster analysis and display of genome-wide expression patterns. Proc. Natl. Acad. Sci. U.S.A. 95:14863-14868

Escaler, M., Aranda, M. A., Thomas, C. L., and Maule, A. J. 2000. Pea embryonic tissues show common responses to the replication of a wide range of viruses. Virology 267:318-325.

Fan, J. B., Yeakley, J. M., Bibikova, M., Chudin, E., Wickham, E., Chen, J., Doucet, D., Rigault, P., Zhang, B., Shen, R., McBride, C., Li, H. R., Fu, X. D., Oliphant, A., Barker, D. L., and Chee, M. S. 2004. A versatile assay for high-throughput gene expression profiling on universal array matrices. Genome Res. 14:878-885.

Fisher, R. A. 1970. Statistical Methods for Research Workers, 14th ed (originally published 1925). Oliver and Boyd, Edinburgh.

Galinsky, V. L. 2003. Automatic registration of microarray images. II. Hexagonal grid. Bioinformatics 19:1832-1836.

Gallie, D. R. 2001. Cap-independent translation conferred by the 5' leader of Tobacco etch virus is eukaryotic initiation factor $4 \mathrm{G}$ dependent. J. Virol. 75:12141-12152.

Gal-On, A., Meiri, E., Elman, C., Gray, D. J., and Gaba, V. 1997. Simple hand-held devices for the efficient infection of plants with viral-encoding constructs by particle bombardment. J. Virol. Methods 64:103-110.

Golem, S., and Culver, J. N. 2003. Tobacco mosaic virus induced alterations in the gene expression profile of Arabidopsis thaliana. Mol. PlantMicrobe Interact. 16:681-688.

Goncalves, P. M., Griffioen, G., Minnee, R., Bosma, M., Kraakman, L. S., Mager, W. H., and Planta, R. J. 1995. Transcription activation of yeast ribosomal protein genes requires additional elements apart from binding sites for Abf1p or Rap1p. Nucleic Acids Res. 23:1475-1480.

Hanley-Bowdoin, L., Settlage, S. B., Orozco, B. M., Nagar, S., and Robertson, D. 2000. Geminiviruses: Models for plant DNA replication, transcription, and cell cycle regulation. Crit. Rev. Biochem. Mol. Biol. 35:105-140.

Huang, Z., Yeakley, J. M., Garcia, E. W., Holdridge, J. D., Fan, J. B., and Whitham, S. A. 2005. Salicylic acid-dependent expression of host genes in compatible Arabidopsis-virus interactions. Plant Physiol. 137:11471159.

Itaya, A., Matsuda, Y., Gonzales, R. A., Nelson, R. S., and Ding, B. 2002. Potato spindle tuber viroid strains of different pathogenicity induces and suppresses expression of common and unique genes in infected tomato. Mol. Plant-Microbe Interact. 15:990-999.

Kasschau, K. D., Xie, Z., Allen, E., Llave, C., Chapman, E. J., Krizan, K. A., and Carrington, J. C. 2003. P1/HC-Pro, a viral suppressor of RNA silencing, interferes with Arabidopsis development and miRNA function. Dev. Cell 4:205-217.

Kenward, M. G., and Roger, J. H. 1997. Small sample inference for mixed effects from restricted maximum likelihood. Biometrics 53:983-997.

Lecellier, C. H., and Voinnet, O. 2004. RNA silencing: No mercy for viruses? Immunol. Rev. 198:285-303.

Lellis, A. D., Kasschau, K. D., Whitham, S. A., and Carrington, J. C. 2002. Loss-of-susceptibility mutants of Arabidopsis thaliana reveal an essential role for eIF(iso)4E during Potyvirus infection. Curr. Biol. 12:1046-1051.

Love, A. J., Yun, B. W., Laval, V., Loake, G. J., and Milner, J. J. 2005. Cauliflower mosaic virus, a compatible pathogen of Arabidopsis, engages three distinct defense-signaling pathways and activates rapid systemic generation of reactive oxygen species. Plant Physiol. 139:935-948.

Mager, W. H., and Planta, R. J. 1991. Coordinate expression of ribosomal protein genes in yeast as a function of cellular growth rate. Mol. Cell. Biochem. 104:181-187.

Mallory, A. C., Reinhart, B. J., Bartel, D., Vance, V. B., and Bowman, L. H. 2002. A viral suppressor of RNA silencing differentially regulates the accumulation of short interfering RNAs and micro-RNAs in tobacco. Proc. Natl. Acad. Sci. U.S.A. 99:15228-15233.

Manly, K. F., Nettleton, D., and Hwang, J. T. 2004. Genomics, prior probability and statistical tests of multiple hypotheses. Genome Res. 14:997-1001.

Maule, A., Leh, V., and Lederer, C. 2002. The dialogue between viruses and hosts in compatible interactions. Curr. Opin. Plant Biol. 5:279-284.

Melaragno, J. E., Mehrotra, B., and Coleman, A. W. 1993. Relationship between endopolyploidy and cell size in epidermal tissue of Arabidopsis.
Plant Cell 5:1661-1668.

Nagar, S., Pedersen, T. J., Carrick, K. M., Hanley-Bowdoin, L., and Robertson, D. 1995. A geminivirus induces expression of a host DNA synthesis protein in terminally differentiated plant cells. Plant Cell 7:705-719.

Padmanabhan, M. S., Goregaoker, S. P., Golem, S., Shiferaw, H., and Culver, J. N. 2005. Interaction of the Tobacco mosaic virus replicase protein with the Aux/IAA protein PAP1/IAA26 is associated with disease development. J. Virol. 79:2549-2558.

Planta, R. J., and Mager, W. H. 1998. The list of cytoplasmic ribosomal proteins of Saccharomyces cerevisiae. Yeast 14:471-477.

Redman, J. C., Haas, B. J., Tanimoto, G., and Town, C. D. 2004. Development and evaluation of an Arabidopsis whole genome Affymetrix probe array. Plant J. 38:545-561.

Sanchez, F., Martinez-Herrera, D., Aguilar, I., and Ponz, F. 1998. Infectivity of Turnip mosaic potyvirus cDNA clones and transcripts on the systemic host Arabidopsis thaliana and local lesion hosts. Virus Res. 55:207-219.

Schoof, H., Ernst, R., Nazarov, V., Pfeifer, L., Mewes, H. W., and Mayer, K. F. 2004. MIPS Arabidopsis thaliana Database (MAtDB): An integrated biological knowledge resource for plant genomics. Nucleic Acids Res. 32:D373-376.

Senthil, G., Liu, H., Puram, V. G., Clark, A., Stromberg, A., and Goodin, M. M. 2005. Specific and common changes in Nicotiana benthamiana gene expression in response to infection by enveloped viruses. J. Gen. Virol. 86:2615-2625.

Storey, J. D., and Tibshirani, R. 2003. Statistical significance for genomewide studies. Proc. Natl. Acad. Sci. U.S.A. 100:9440-9445.

Tusher, V. G., Tibshirani, R., and Chu, G. 2001. Significance analysis of microarrays applied to the ionizing radiation response. Proc. Natl. Acad. Sci. U.S.A. 98:5116-5121.

Ulm, R., and Nagy, F. 2005. Signaling and gene regulation in response to ultraviolet light. Curr. Opin. Plant Biol. 8:477-482.

Ulm, R., Baumann, A., Oravecz, A., Mate, Z., Adam, E., Oakeley, E. J., Schafer, E., and Nagy, F. 2004. Genome-wide analysis of gene expression reveals function of the bZIP transcription factor HY5 in the UV-B response of Arabidopsis. Proc. Natl. Acad. Sci. U.S.A. 101:1397-1402.

Venema, J., and Tollervey, D. 1999. Ribosome synthesis in Saccharomyces cerevisiae. Annu. Rev. Genet. 33:261-311.

Voinnet, O. 2001. RNA silencing as a plant immune system against viruses. Trends Genet. 17:449-459.

Wang, D., and Maule, A. J. 1995. Inhibition of host gene expression associated with plant virus replication. Science 267:229-231.

Whitham, S. A. 2004. Viral Host Genomics. Pages 1269-1272 in: Encyclopedia of Plant and Crop Science. R. M. Goodman, ed. Marcel Dekker, Inc., New York.

Whitham, S. A., and Wang, Y. 2004. Roles for host factors in plant viral pathogenicity. Curr. Opin. Plant Biol. 7:365-371.

Whitham, S. A., Quan, S., Chang, H. S., Cooper, B., Estes, B., Zhu, T., Wang, X., and Hou, Y. M. 2003. Diverse RNA viruses elicit the expression of common sets of genes in susceptible Arabidopsis thaliana plants. Plant J. 33:271-283.

Whitham, S. A., Yang, C., and Goodin, M. M. 2006. Global impact: Elucidating plant responses to viral infection. Mol. Plant-Microbe Interact. 19:1207-1215.

Wolfinger, R. D., Gibson, G., Wolfinger, E. D., Bennett, L., Hamadeh, H., Bushel, P., Afshari, C., and Paules, R. S. 2001. Assessing gene significance from cDNA microarray expression data via mixed models. J. Comput. Biol. 8:625-637.

Zeenko, V., and Gallie, D. R. 2005. Cap-independent translation of Tobacco etch virus is conferred by an RNA pseudoknot in the $5^{\prime}$-leader. $\mathrm{J}$. Biol. Chem. 280:26813-26824.

Zhu, S., Gao, F., Cao, X., Chen, M., Ye, G., Wei, C., and Li, Y. 2005. The Rice dwarf virus $\mathrm{P} 2$ protein interacts with ent-kaurene oxidases in vivo, leading to reduced biosynthesis of gibberellins and rice dwarf symptoms. Plant Physiol. 139:1935-1945.

\section{AUTHOR-RECOMMENDED INTERNET RESOURCES}

EMBL-European Bioinformatics Institute ArrayExpress database: www.ebi.ac.uk

Vienna University of Economics and Business Administration Department of Statistics and Mathematics R project for statitstical computing: www.r-project.org

The Arabidopsis information resource (TAIR) Gene Ontology website: www.arabidopsis.org/tools/bulk/go/index.jsp

MIPS functional classification system: mips.gsf.de/proj/funcatDB/search_main_frame.html 